\title{
Uso do código SWAT na previsão de vazão dos rios da bacia hidrográfica Tocantins-Araguaia
}

\section{Use of the SWAT code in the ground forecast of rivers of the Tocantins-Araguaia hydrographic bowl}

Ricardo Deus - Doutorado em Química com ênfase em Modelagem Ambiental Integrada, pela Universidade Federal do Pará (UFPA). Doutorado complementar no Departamento de Engenharia do Ambiente da Universidade Técnica de Lisboa (UTL-Portugal). Professor da Universidade Federal do Pará (UFPA). E-mail: dedeus@ufpa.br

Simonny Simões Deus - Doutoranda do Programa de Pós-Graduação em Química da Universidade Federal do Pará (UFPA). E-mail: simonny@ufpa.br

Ramiro Joaquim de Jesus Neves - Doutorado em Ciências Aplicadas pela Université de Liège. Professor associado do Instituto Superior Técnico (UTL), Portugal. E-mail: ramiro.neves@ist.utl.pt

\section{Resumo}

A bacia hidrográfica Tocantins-Araguaia é caracterizada por apresentar singularidade na ocorrência de enchentes e deslizamentos de massa em função de seus altos valores de vazão. Desse modo, o objetivo foi aplicar o simulador Soil and Water Assessment Tool (SWAT) para promover a previsão de vazão dos rios da bacia hidrográfica Tocantins-Araguaia e criar cenários para avaliação do papel da dinâmica da taxa de fluxo em função das altas precipitações e desmatamento. O modelo apresentou valores aceitáveis na calibração e validação e posteriormente, cenários de 0 a $100 \%$ da mudança do uso da terra foram simulados. Os resultados mostram que com perda de $10 \%$ da vegetação na bacia durante períodos de alto escoamento pluviométrico, o escoamento superficial e a erosão aumentam, causando problemas de sedimentação e inundação. Assim, o modelo possibilitou uma compreensão abrangente do sistema para o desenvolvimento da melhor política de manejo e tomada de decisão.

\section{Palavra-chave}

Modelagem. Enchente. Desmatamento. Gestão Ambiental.

\begin{abstract}
The Tocantins-Araguaia watershed is characterized by its singularity in the occurrence of floods and landslides due to its high flow rates. Thus, the objective was to apply the Soil and Water Assessment Tool (SWAT) simulator to promote the flow forecast of rivers in the Tocantins-Araguaia hydrographic basin and create scenarios for assessing the role of flow rate dynamics due to high precipitation and deforestation. The model presented acceptable values in calibration and validation and subsequently, scenarios from 0 to $100 \%$ of land use change were simulated. The results show that with $10 \%$ loss of vegetation in the basin during periods of high rainfall, surface runoff and erosion increase, causing sedimentation and flooding problems. Thus, the model enabled a comprehensive understanding of the system to develop the best management and decision-making policy.
\end{abstract}

\section{Keywords}

Modeling. Flood. Deforestation. Environmental Management. 


\section{INTRODUÇÃO}

As bacias hidrográficas podem ser consideradas como a unidade básica de planejamento de ecossistemas, onde se observa a delicada relação de interdependência dos fatores bióticos e abióticos, terrestres e aquáticos, sendo que perturbações antrópicas podem comprometer a dinâmica de seu funcionamento (NOGUEIRA et al., 2010). Neste sentido, a bacia hidrográfica dos rios Tocantins e Araguaia, compreendida em parte no Centro-Oeste e no Norte do Brasil, foi definida pela Agência Nacional de Águas (ANA) como uma das regiões prioritárias para implementação dos dispositivos da nova Política Nacional de Recursos Hídricos, para a qual, em função dos múltiplos empreendimentos em execução e projetados; e dos potenciais conflitos socioambientais que apresenta, deseja-se elaborar um Plano de Bacia com caráter estratégico, que permita estabelecer diretrizes para a compatibilização do uso múltiplo dos recursos hídricos (abastecimento humano, geração de energia, navegação, irrigação, entre outros) com as demais políticas setoriais que tenham interferência sobre os recursos hídricos e com a preservação ambiental, para que o desenvolvimento promovido seja sustentável (ANA, 2005).

Contudo, uma questão que necessita ser avaliada com critério científico é a relação entre extensão da bacia hidrográfica Tocantins-Araguaia e particularidades relacionadas à altitude, meteorologia, uso e tipo de solo, desde cotas próximas a $1.000 \mathrm{~m}$ na região Centro-Oeste (nascente) até cerca de $70 \mathrm{~m}$ ao Norte (foz), dadas algumas regiões localizadas em altitudes mais baixas que enfrentam intensa atividade erosiva provocando assoreamentos, favorecendo a ocorrência de enchentes e causando alterações ecológicas (RAIOL, 2010). Como exemplo, temos a região onde se localiza a cidade de Marabá (maior polo siderúrgico da região Norte) e a Usina Hidrelétrica de Tucuruí (UHT) - segunda maior hidrelétrica do país, com produção de 8 milhões de KW, equivalente a $32 \%$ do potencial total da bacia -, as quais enfrentam fortes enchentes e riscos de assoreamento, respectivamente, em períodos de alta precipitação (ANEEL, 2002; MMA, 2006; PROJETO AVA MARABÁ, 2006; PREFEITURA MUNICIPAL DE MARABÁ, 2010; ELETROBRÁS, 2011).

Diante deste contexto, uma pesquisa realizada dentro de uma bacia hidrográfica deve envolver medições de todas as variáveis que influenciam sua dinâmica. Esta é uma tarefa difícil, por vezes impossível, pois o tempo necessário e os custos de medição e monitoramento são os principais obstáculos (MACHADO, 2002). Devido a isso, os modelos matemáticos estão sendo cada 
vez mais usados para os estudos de bacias. Dentre estes, destaca-se o código SWAT (Soil and Water Assessment Too), que é um software de domínio público desenvolvido em 1996 nos Estados Unidos pelo Serviço de Pesquisa Agrícola (ARS) e pela Universidade do Texas, o qual incorpora avanços de modelos já existentes. Além disso, este modelo é uma ferramenta eficaz para apoiar a gestão de recursos hídricos em ampla gama de escalas e condições ambientais em todo o globo (ARNOLD et al., 1998; ARNOLD; FOHRER, 2005; GASSMAN et al., 2007). Os dados de precipitação são tipicamente o insumo mais importante para os modelos hidrológicos e, portanto, precisa-se de dados que descrevam a variabilidade espacial e temporal da precipitação para iniciar modelagem hidrológica e gestão da bacia hidrográfica (STRAUCH et al., 2011). Desse modo, o objetivo foi aplicar o simulador SWAT para promover a previsão da vazão dos rios da bacia hidrográfica Tocantins-Araguaia e criar cenários para avaliação do papel da dinâmica de fluxo em função das altas precipitações e desmatamento.

\section{MATERIAL E MÉTODOS}

\section{1 ÁREA DE ESTUDO}

A área de estudo está delimitada a partir da nascente da bacia hidrográfica Tocantins-Araguaia até a barragem da Usina Hidrelétrica de Tucuruí (UHT) (exutório), conforme a Figura 1. Ainda na Figura 1 é possível localizar os pontos de monitoramento MP1, MP2 e MP3 simulados pelo SWAT, próximo ao seu exutório: o ponto MP1 corresponde ao conjunto de contribuição dos rios Tocantins, Araguaia e Itacaiúnas; o ponto MP2 representa os rios principais da bacia (Tocantins e Araguaia); e o ponto MP3 refere-se ao principal afluente (rio Itacaiúnas). Além disso, na Figura 1 são representadas as localizações de 15 estações de monitoramento como base de dados para construção dos modelos no referido estudo (Brasília - BRA, Marabá - MARA, Imperatriz - IMPE, Araguaia - ARAG, Carolina - CARO, Conceição do Araguaia - CONA, Pedro Afonso - PEDA, Alto do Paraíba - ALTP, Porto Nacional - PORN, Peixes - PEIX, Taguatinga TAGU, São Felix do Araguaia - SFAR, Fazenda Piratininga - FPIR, São Miguel do Araguaia - SMRA, Santa Fé - SAFE), disponíveis na Agência Nacional de Águas (ANA) pelo Sistema de Informações Hidrológicas (HIDROWEB) (ANA, 2020). A bacia hidrográfica Tocantins-Araguaia possui uma grande área de captação, estendida entre os paralelos $2^{\circ} \mathrm{S}$ e $18^{\circ} \mathrm{S}$ e os meridianos $46^{\circ} \mathrm{W}$ e $56^{\circ} \mathrm{W}$, com área de drenagem de $767.000 \mathrm{~km}^{2}$ (ANEEL, 2002), constituindo a maior 
bacia hidrográfica inteiramente dentro do território brasileiro, incluindo grandes cidades, atividades agrícolas, mineração e usinas de energia elétrica, distribuídas nos estados de Goiás, Tocantins, Pará, Maranhão, Mato Grosso e Distrito Federal (Figura 1). A área de drenagem inclui $343.000 \mathrm{~km}^{2}$ correspondentes ao rio Tocantins, $382.000 \mathrm{~km}^{2}$ ao rio Araguaia (seu principal afluente) e $42.000 \mathrm{~km}^{2}$ ao rio Itacaiúnas (o maior contribuinte de seu curso inferior) (ANEEL, 2002). A bacia do Tocantins-Araguaia possui uma vazão média de $11.000 \mathrm{~m}^{3} \mathrm{~s}^{-1}$, com contribuição do rio Tocantins (40\%), do rio Araguaia (45\%) e do rio Itacaiúnas (5\%) (ANEEL, 2002).

Figura 1 - Projeto ArcSWAT da bacia hidrográfica Tocantins-Araguaia, limitado à barragem da Usina Hidrelétrica de Tucuruí (UHT)

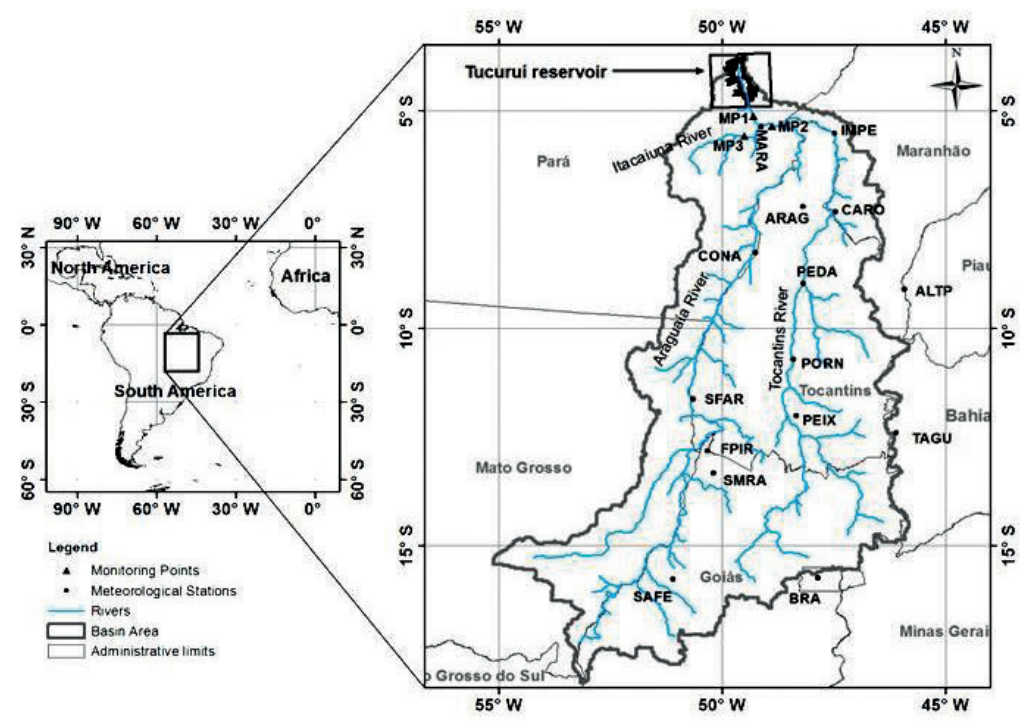

Fonte: Autores.

De acordo com a Secretaria de Recursos Hídricos do Ministério do Meio Ambiente (MMA, 2006), o clima da região hidrográfica é tropical, com temperatura anual média de $26^{\circ} \mathrm{C}$, e dois períodos climáticos bem definidos: o chuvoso, de outubro a abril, com mais de $90 \%$ da precipitação; e o seco, de maio a setembro, com baixa umidade relativa do ar. A quantidade de precipitação na bacia é maior em sua porção Norte, mais próxima a sua foz, diminuindo ao CentroOeste, na região de suas nascentes, com uma variação de sua média anual de 1.580 a 2.300mm (ANA, 2004). Segundo dados da ANA (2004), a precipitação média da bacia Tocantins-Araguaia é de ordem de $1.600 \mathrm{~mm} /$ ano, a vazão é de 
$11.800 \mathrm{~m}^{3}$ /s (ANEEL, 2002), fornecendo uma vazão específica média de 15,6L/s. $\mathrm{km}^{2}$, uma evapotranspiração real média de $1.200 \mathrm{~mm} /$ ano e um coeficiente médio de escoamento superficial de aproximadamente 0,30 (ANA, 2004). A região possui dois biomas principais: a floresta amazônica, que ocupa a região norte/noroeste (35\% da área total), e a savana tropical (65\%). Esses biomas incluem flora e fauna diversas e uma ampla zona de transição de habitat (ecótono) (ANA, 2009).

\subsection{CONFIGURANDO O MODELO}

\subsubsection{Modelo Digital de Elevação do Terreno (DEM)}

A topografia da bacia hidrográfica Tocantins-Araguaia foi definida por um Modelo Digital de Elevação (DEM), que descreveu a elevação de pontos em uma determinada área com uma resolução espacial específica de $3 \mathrm{~km}$ por $3 \mathrm{~km}$. Criado o DEM, foi importado para o formato de grade Arcview com "UTM - Zona $20 \mathrm{~S}$ projeção WGS84 Datum”. O DEM foi utilizado para delinear o divisor de águas e analisar os padrões de drenagem do terreno da superfície da terra. Tamanho e número das sub-bacias são determinados de acordo com as correntes. O mapa da grade de uso da terra foi preparado para usar imagem do mapa de dados citados na literatura (ÖZTÜRK et al., 2008).

Foram utilizadas cartas de tipo de solo e uso de solo (1:200.000) em arquivo no formato "shp", as quais foram fornecidas pelo Instituto Brasileiro de Geografia e Estatística (IBGE), bem como uma descrição do perfil de textura para todos os solos. Para cada tipo de solo, a porcentagem de solo de argila, silte, areia, e o percentual de matéria orgânica (OAKES, 1954; ONALP, 1982; POLAT, 2000; ESWARAN et al., 2003; ESBN, 2005), foram utilizadas duas configurações de camada de solos: coluna 1, camada superficial com espessura de $40 \mathrm{~cm}$; e Coluna 2, camada subsuperficial com espessura de $80 \mathrm{~cm}$. Todos estes dados foram inseridos no banco de dados SWAT/2005 manual ou em formato "dbf".

$\mathrm{Na}$ aplicação do modelo SWAT, a bacia foi dividida em sub-bacias, cujo tamanho dependeu diretamente do valor limite (CSTV) obtido. O limiar é um parâmetro importante para a definição das Unidades de Resposta Hidrológica (em inglês HRU) e para permitir a divisão de sub-bacia mais detalhadas (FITZHUGH; MACKAY, 2000; ROMANOWICZ et al., 2005). No total, obtiveram-se 109 sub-bacias hidrográficas e 1.969 HRU sendo definidas utilizando as rotinas de delimitação automática. As HRU foram caracterizadas pelas classes de uso do solo. 


\subsection{PREPARAÇÃO DE DADOS DO CLIMA}

Os dados simulados foram obtidos em séries temporais de precipitação diária, temperatura do ar, umidade relativa do ar máxima e mínima, velocidade do vento e radiação solar, disponibilizados a partir de 15 estações meteorológicas distribuídas na bacia do Tocantins-Araguaia (Tabela 1), administradas pelo Instituto Nacional de Meteorologia (INMET-Brasil) e pela Agência Nacional de Águas (ANA), no período de janeiro de 2007 a dezembro de 2010.

Tabela 1 - Especificações das estações meteorológicas utilizadas para a produção do modelo

\begin{tabular}{|c|c|c|c|c|c|c|c|c|}
\hline ID & 竞 & io &  & 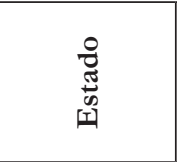 & 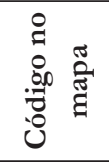 &  & 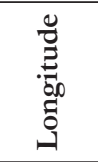 & 苞 \\
\hline 1 & INMET & 01547004 & Brasília & $\begin{array}{c}\text { Distrito } \\
\text { Federal (DF) }\end{array}$ & BRA & -15.74 & -47.86 & 1061 \\
\hline 2 & INMET & 82562 & Marabá & Pará (PA) & MARA & -5.36 & -49.13 & 117 \\
\hline 3 & INMET & 82564 & Imperatriz & $\begin{array}{c}\text { Maranhão } \\
\text { (MA) }\end{array}$ & IMPE & -5.53 & -47.48 & 211 \\
\hline 4 & INMET & 82659 & Araguaína & $\begin{array}{c}\text { Tocantins } \\
\text { (TO) }\end{array}$ & ARAG & -7.20 & -48.20 & 351 \\
\hline 5 & INMET & 82765 & Carolina & $\begin{array}{c}\text { Maranhão } \\
\text { (MA) }\end{array}$ & CARO & -7.33 & -47.46 & 448 \\
\hline 6 & INMET & 82861 & $\begin{array}{c}\text { Conceição } \\
\text { do Araguaia }\end{array}$ & Pará (PA) & CONA & -8.26 & -49.26 & 182 \\
\hline 7 & INMET & 82863 & $\begin{array}{c}\text { Pedro } \\
\text { Afonso }\end{array}$ & $\begin{array}{c}\text { Tocantins } \\
\text { (TO) }\end{array}$ & PEDA & -8.96 & -48.18 & 240 \\
\hline 8 & INMET & 82970 & $\begin{array}{c}\text { Alto } \\
\text { Parnaíba }\end{array}$ & $\begin{array}{c}\text { Maranhão } \\
\text { (MA) }\end{array}$ & ALTP & -9.1 & -45.93 & 481 \\
\hline 9 & INMET & 83064 & $\begin{array}{c}\text { Porto } \\
\text { Nacional }\end{array}$ & $\begin{array}{c}\text { Tocantins } \\
\text { (TO) }\end{array}$ & PORN & -10.71 & -48.41 & 302 \\
\hline 10 & INMET & 83228 & Peixes & $\begin{array}{c}\text { Tocantins } \\
(\mathrm{TO})\end{array}$ & PEIX & -12.01 & -48.35 & 304 \\
\hline 11 & INMET & 83235 & Taguatinga & $\begin{array}{c}\text { Tocantins } \\
(\mathrm{TO})\end{array}$ & TAGU & -12.4 & -46.10 & 635 \\
\hline 12 & ANA & 26350000 & $\begin{array}{c}\text { São Félix do } \\
\text { Araguaia }\end{array}$ & $\begin{array}{c}\text { Mato } \\
\text { Grosso } \\
\text { (MT) }\end{array}$ & SFAR & -11.62 & -50.66 & 190 \\
\hline 13 & ANA & 01250000 & $\begin{array}{c}\text { Fazenda } \\
\text { Piratininga }\end{array}$ & Goiás (GO) & FPIR & -12.82 & -50.34 & 220 \\
\hline 14 & ANA & 01350002 & $\begin{array}{l}\text { São Miguel } \\
\text { do Araguaia }\end{array}$ & Goiás (GO) & SMRA & -13.33 & -50.19 & 249 \\
\hline 15 & ANA & 01551003 & Santa Fé & Goiás (GO) & SAFE & -15.77 & -51.10 & 379 \\
\hline
\end{tabular}

Fonte: HIDROWEB (ANA, 2020). 


\subsection{SENSIBILIDADE DO MODELO}

Os dados de fluxos disponíveis para analisar a sensibilidade foram medidos de janeiro de 2007 a dezembro de 2008, em três pontos de monitoramento (MP1, MP2, MP3), localizados dentro dos principais afluentes da bacia. O período de validação foi realizado utilizando os dados nos mesmos locais, durante o período de janeiro de 2009 a dezembro de 2010. O método de análise de sensibilidade implementado no modelo SWAT é chamado de Latin Hypercube One-factor-At-aTime (LH-OAT) proposto por Morris (1991) e Van Griensven et al. (2006), pois esta função implementa o procedimento de fator único como método de triagem.

\subsection{CALIBRAÇÃO E VALIDAÇÃO DO MODELO}

A calibração automática foi usada para identificar um conjunto de parâmetros do modelo otimizando uma boa estatística de ajuste entre os valores observados e previstos para posterior validação automatizada, mesma técnica usada de comparação com período distinto. A calibração e a validação manual foram obtidas por meio de cálculos estatísticos. Neste estudo, foram calculados, de acordo com Pisinaras et al. (2010): o quadrado do coeficiente de correlação $\left(\mathrm{R}^{2}\right)$, o erro quadrático médio (RMSE), a função normalizada objetiva (NOF) e diagramas de dispersão.

O erro quadrático médio (RMSE) e da função objetivo normalizada (NOF) (KORNECKI; SABBAGH; STORM, 1999; HESSION et al., 1994) foram calculados com base nas seguintes equações (Eq.1 e Eq.1.1):

$$
\begin{aligned}
& \text { RMSE }=\sqrt{\frac{\sum_{i=1}^{\mathrm{N}}\left(\mathrm{P}_{i}-\mathrm{O}_{i}\right)^{2}}{\mathrm{~N}}} \\
& \text { NOF }=\frac{\text { RMSE }}{\overline{0}}
\end{aligned}
$$

Onde "Pi" são os valores previstos do modelo, "Oi" são os valores observados para as observações "N", e “ō" é a média dos valores observados. De acordo com Kornecki, Sabbagh e Storm (1999), o valor ideal da NOF é 0,0 . No entanto, um modelo aceitável para valores NOF no intervalo de 0,0 a 1,0, quando os dados específicos do local estão disponíveis para a calibração. Segundo Pisinaras et al. (2010), o modelo pode ser utilizado para testar os cenários associados com as práticas de gestão. 
Outra forma de avaliar o modelo foi por meio da utilização de diagramas de dispersão (TSIHRINTZIS; HAMID, 1998; TSIHRINTZIS; SIDAN, 1998), onde as quantidades previstas foram traçadas contra aqueles observados. Segundo Pisinaras et al. (2010), em um diagrama de dispersão, uma linha reta de regressão da seguinte forma é também equipado com os dados:

$\mathrm{Pi}=\gamma$ Oi, onde o valor da inclinação $(\gamma)$ é uma medida de correlação positiva (se $\gamma>1,0)$ ou negativa ( $\operatorname{se} \gamma<1,0)$ no âmbito de previsão do modelo em comparação com os dados observados. Além disso, o quadrado do coeficiente de correlação " $\mathrm{R}$ "” da linha de regressão é calculado. Quanto mais baixo de 1,0 for o valor de $\mathrm{R}^{2}$, pior a correlação de dados existentes, ou seja, maior será a dispersão dos dados em torno da linha. Portanto, uma melhor calibração requer que os

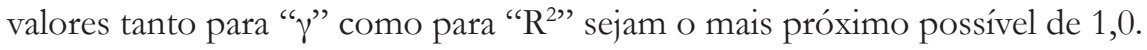

A validação e a calibração automática dos três pontos de monitoramento foram comparadas com a calibração e a validação manual.

\subsection{MODELOS DE VAZÃO E CENÁRIOS DE PREVISÃO DE VAZÃO DOS RIOS COM BASE EM MUDANÇAS NO USO DA TERRA}

Após a calibração e a validação do modelo, a simulação foi realizada com dados de precipitação e vazão, por um período de quatro anos para comprovar a influência da precipitação sobre a vazão da bacia hidrográfica e, posteriormente, feita a comparação dos três pontos de monitoramento. Além disso, três cenários foram criados: o primeiro simula a vazão da bacia a partir de dados reais; os outros dois simulam a previsão de vazão dos rios da bacia Tocantins-Araguaia em função das mudanças de uso da terra, como, por exemplo, a retirada da cobertura vegetal da margem da bacia em 10\% e 50\%, respectivamente. Em seguida foi realizada a comparação entre eles. Estas mudanças que ocorrem nas condições naturais de vegetação e solo, onde as propriedades se comprimem, impedem a infiltração de água e, assim, aumentam o fluxo de água em rios (ZACHAR, 1982; PRIMAVESI, 1987; BERTONI; LOMBARDI NETO, 1990).

\section{RESULTADOS}

\subsection{MODELO DE PREVISÃO DE VAZÃO}

Com base no Modelo Digital de Elevação do Terreno, foi possível observar que a declividade aumenta a velocidade do fluxo de água no sentido norte da bacia (Figura 2). 
Figura 2 - Modelo Digital de Elevação para bacia hidrográfica Tocantins-Araguaia, limitado à barragem da Usina Hidrelétrica de Tucuruí (UHT)



Fonte: Autores.

A partir da divisão da bacia em sub-bacias e HRU (Figura 3), obtiveram-se características mais realísticas sobre a região de estudo, no que se refere ao uso e ao tipo do solo.

Figura 3 - Bacia hidrográfica do Tocantins-Araguaia, subdividida em 109 subbacias, contendo pontos de monitoramento, estações meteorológicas, uso e tipo de solo

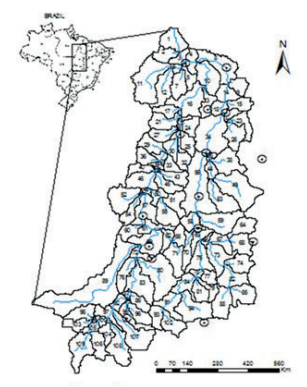

Legenda

$\square$ Bacia Hidrográfica Drenagem

○ Pontos de Monitoramento e/ou Estacões Meteorológicas

Fonte: Autores.

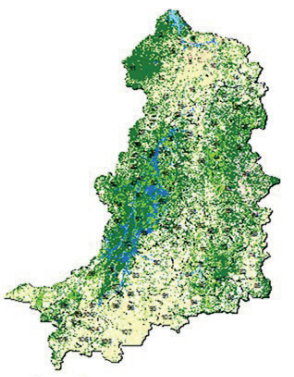

Legenda

Uso do Solo

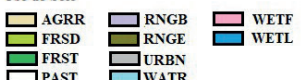

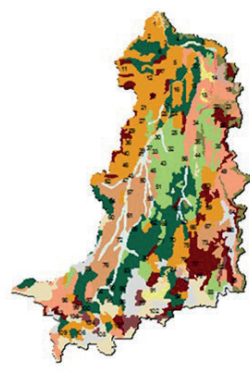

Legenda

Tipo de Solo

Afloramentos de Roch

Cambissolo Háplico Dunas

$\square$ Plintossolo Háplico

Gleissolo Háplico

Latossolo Amarelo

Latossolo Vermeliso

ArgissoloVermelho

Neossolo Regolitico

Vertissolo Cromado

Latolosso Vermelho-Amarelo Argissolo Vermelho-Amarelo Chemossolo Argilúvico 
Vele destacar maior percentual de floresta mista, seguida de agricultura e pastagem, conforme a Tabela 2, associado às características do tipo de solo de maior evidência na região (Figura 03), como Latossolos e Argissolos: (Amarelo, Vermelho e Vermelho-Amarelo), com texturas variáveis de média a argilosa e arenosos, eventualmente em composição a outros solos.

Tabela 2 - Dados de uso do solo na bacia hidrográfica Tocantins-Araguaia

\begin{tabular}{l|c|c}
\hline \multicolumn{1}{c|}{ Uso do solo } & $\begin{array}{c}\text { Código SWAT } \\
\text { (em inglês) }\end{array}$ & $\begin{array}{c}\text { Percentagem na bacia } \\
\mathbf{( \% )}\end{array}$ \\
\hline Floresta mista & FRST & 36.12 \\
\hline Terras agrícolas & AGRR & 33.10 \\
\hline Pastagem & PAST & 20.07 \\
\hline Floresta decídua & FRSD & 5.72 \\
\hline Pantanais mistos & WETL & 2.67 \\
\hline Plantas forrageiras & RNGB & 0.94 \\
\hline Água & WATR & 0.90 \\
\hline Pantanais florestados & WETF & 0.46 \\
\hline Gramíneas & RNGE & 0.01 \\
\hline Áreas urbanas & URBN & 0.01 \\
\hline
\end{tabular}

Fonte: Autores.

Os testes de sensibilidade do modelo foram importantes para identificar os parâmetros que apresentaram melhores respostas e assim foram selecionados para calibração e validação do modelo (Tabela 3).

Tabela 3 - Parâmetros usados na calibração e validação do modelo SWAT

\begin{tabular}{|c|c|c|c|c|c|}
\hline Variável & $\begin{array}{c}\text { Processos } \\
\text { do modelo }\end{array}$ & Descrição & $\begin{array}{c}\text { Intervalo } \\
\text { de faixa }\end{array}$ & $\begin{array}{l}\text { Valores } \\
\text { usados }\end{array}$ & Referências \\
\hline $\mathrm{CN} 2$ & $\begin{array}{c}\text { Escoamento } \\
\text { superficial }\end{array}$ & $\begin{array}{l}\text { Curva de } \\
\text { número }\end{array}$ & $0-100$ & 82 & $\begin{array}{l}\text { This Study: Arnold et } \\
\text { al., 1998; Neitsh et al., } \\
\text { 2005; Bouraoui et al., } \\
\text { 2005. }\end{array}$ \\
\hline ESCO & $\begin{array}{c}\text { Evapotran- } \\
\text { piração }\end{array}$ & $\begin{array}{l}\text { Fator de com- } \\
\text { pensação da } \\
\text { evaporação do } \\
\text { solo }\end{array}$ & $0.0-1.0$ & 0.95 & $\begin{array}{l}\text { Farida Dechmi et al., } \\
\text { 2012; Muhammed-Er- } \\
\text { nur Akiner e Atila Ak- } \\
\text { koyunlu, 2012; Arnold } \\
\text { et al.,1998; Neitsh et al., } \\
\text { 2005; Bouraoui et al., } \\
\text { 2005; Michael Strauch } \\
\text { et al., 2012. }\end{array}$ \\
\hline EPCO & $\begin{array}{c}\text { Água } \\
\text { subterrânea }\end{array}$ & \begin{tabular}{|} 
Fator de com- \\
pensação de \\
absorção pelas \\
plantas
\end{tabular} & $0.0-1.0$ & 1.0 & $\begin{array}{l}\text { This Study: Arnold et } \\
\text { al., 1998; Neitsh et al., } \\
\text { 2005; Bouraoui et al., } \\
2005 \text {. }\end{array}$ \\
\hline
\end{tabular}


continuação

\begin{tabular}{|c|c|c|c|c|c|}
\hline Variável & $\begin{array}{l}\text { Processos } \\
\text { do modelo }\end{array}$ & Descrição & $\begin{array}{l}\text { Intervalo } \\
\text { de faixa }\end{array}$ & $\begin{array}{l}\text { Valores } \\
\text { usados }\end{array}$ & Referências \\
\hline $\begin{array}{lll}\mathrm{S} & \mathrm{O} & \mathrm{L} \\
\text { AWC } & \end{array}$ & $\begin{array}{l}\text { Água no } \\
\text { solo }\end{array}$ & $\begin{array}{l}\text { Avaliação da } \\
\text { capacidade de } \\
\text { água dispo- } \\
\text { nível da ca- } \\
\text { mada de solo } \\
\text { (mmH } \mathrm{m}_{2} \mathrm{O} / \\
\text { mmsolo) } \\
\end{array}$ & $0.0-1.0$ & $\begin{array}{c}0.10- \\
0.16\end{array}$ & $\begin{array}{l}\text { This Study: Arnold et } \\
\text { al., 1998; Neitsh et al., } \\
\text { 2005; Bouraoui et al., } \\
\text { 2005. }\end{array}$ \\
\hline $\begin{array}{l}\text { GW_RE- } \\
\text { VAP }\end{array}$ & $\begin{array}{c}\text { Água } \\
\text { subterrânea }\end{array}$ & \begin{tabular}{|l|} 
Águas \\
subterrâneas- \\
coeficiente \\
"Revap" \\
\end{tabular} & $0.02-0.20$ & 0.02 & $\begin{array}{l}\text { This Study: Arnold et } \\
\text { al., 1998; Neitsh et al., } \\
\text { 2005; Bouraoui et al., } \\
\text { 2005. }\end{array}$ \\
\hline $\begin{array}{l}\text { GW_DE- } \\
\text { LAY }\end{array}$ & $\begin{array}{c}\text { Água } \\
\text { subterrânea }\end{array}$ & $\begin{array}{l}\text { Tempo de } \\
\text { atraso das } \\
\text { águas subter- } \\
\text { râneas }\end{array}$ & $0-50$ & 50 & $\begin{array}{l}\text { Muhammed Ernur } \\
\text { Akiner e Atila Ak- } \\
\text { koyunlu, 2012; Arnold } \\
\text { et al., 1998; Neitsh et } \\
\text { al., 2005; Bouraoui et } \\
\text { al., 2005. }\end{array}$ \\
\hline CN_N2 & $\begin{array}{c}\text { Escoamento } \\
\text { Superficial }\end{array}$ & $\begin{array}{l}\text { Armazena- } \\
\text { mento de "n" } \\
\text { valores para o } \\
\text { canal principal } \\
\end{array}$ & $0.01-0.5$ & 0.05 & $\begin{array}{l}\text { This Study: Arnold et } \\
\text { al., 1998; Neitsh et al., } \\
\text { 2005; Bouraoui et al., } \\
\text { 2005. }\end{array}$ \\
\hline$\overline{\text { GWQMN }}$ & $\begin{array}{c}\text { Água } \\
\text { subterrânea }\end{array}$ & $\begin{array}{l}\text { Limiar da pro- } \\
\text { fundidade da } \\
\text { água no aquí- } \\
\text { fero superfi- } \\
\text { cial necessária } \\
\text { para fluxo de } \\
\text { retorno ocor- } \\
\text { rer }\left(\mathrm{mmH}_{2} \mathrm{O}\right)\end{array}$ & $0.0-300.0$ & 100.0 & $\begin{array}{l}\text { This Study: Arnold et } \\
\text { al., 1998; Neitsh et al., } \\
\text { 2005; Bouraoui et al., } \\
\text { 2005. }\end{array}$ \\
\hline $\begin{array}{l}\mathrm{RCHRG}_{-} \\
\mathrm{DP}\end{array}$ & $\begin{array}{c}\text { Água } \\
\text { subterrânea }\end{array}$ & $\begin{array}{l}\text { Fração de } \\
\text { percolação no } \\
\text { aquífero pro- } \\
\text { fundo } \\
\end{array}$ & $0.0-1.0$ & $0.0-0.8$ & $\begin{array}{l}\text { This Study: Arnold et } \\
\text { al., 1998; Neitsh et al., } \\
\text { 2005; Bouraoui et al., } \\
\text { 2005. }\end{array}$ \\
\hline $\begin{array}{l}\text { ALPHA_ } \\
\text { BF }\end{array}$ & $\begin{array}{c}\text { Água } \\
\text { subterrânea }\end{array}$ & $\begin{array}{l}\text { Fator alfa de } \\
\text { base de fluxo } \\
\text { (dias) }\end{array}$ & $0.0-1.0$ & 0.1 & $\begin{array}{l}\text { This Study: Arnold et } \\
\text { al., 1998; Neitsh et al., } \\
\text { 2005; Bouraoui et al., } \\
\text { 2005. }\end{array}$ \\
\hline
\end{tabular}

Fonte: Autores.

O modelo foi calibrado e validado com base na taxa de vazão, utilizando dados dos três pontos de monitoramento (MP1, MP2, MP3), localizados próximos ao exutório da bacia. Após a obtenção de uma calibração bem sucedida, realizada com uma série de dados obtidos em dois anos (janeiro de 2007 a dezembro de 2008), mantendo-se este dois anos de dados e acrescentando mais dois anos 
(janeiro de 2009 a dezembro de 2010), o modelo foi validado e comparado com os valores de vazão observados (Figuras 4, 5 e 6 e Tabela 4). No período de calibração da vazão média simulada no ponto de monitoramento MP1 foi obtido o valor médio $13,419 \mathrm{~m}^{3} \mathrm{~s}^{-1}$ sendo comparado com o valor da média da vazão a partir de dados de campo que foi $13,258 \mathrm{~m}^{3} \mathrm{~s}^{-1}$ (Figura 4).

Figura 4 - Medição e simulação de vazões e diagrama de dispersão correspondente ao ponto de monitoramento MP1 para calibração e validação

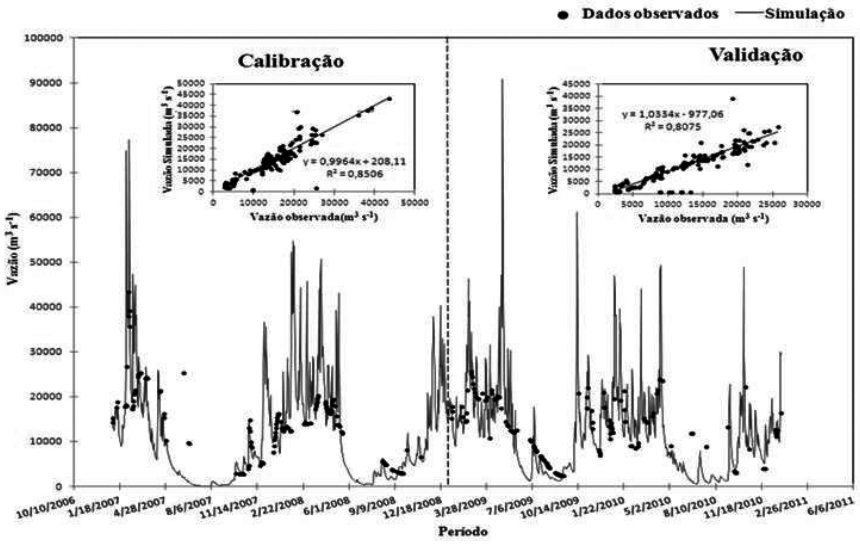

Fonte: Autores.

A simulação média do ponto MP2 foi de $5,738 \mathrm{~m}^{3} \mathrm{~s}^{-1}$. Foi feita também a comparação com o valor da média da vazão a partir de dados de campo que foi $6,010 \mathrm{~m}^{3} \mathrm{~s}^{-1}$ (Figura 5).

Figura 5 - Medição e simulação de vazões e diagrama de dispersão correspondente ao ponto de monitoramento MP2 para calibração e validação

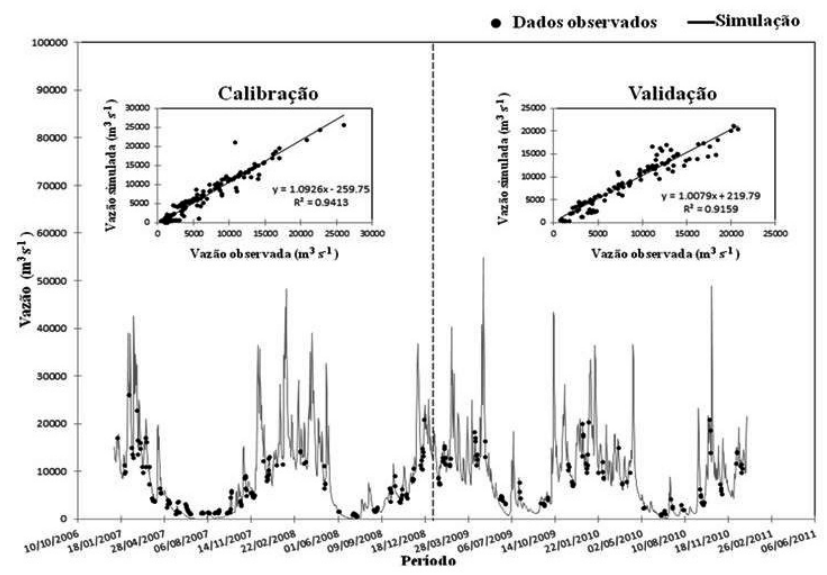

Fonte: Autores. 
A vazão média simulada no ponto de monitoramento MP3 foi $434 \mathrm{~m}^{3} \mathrm{~s}^{-1}$ enquanto que o valor da média da vazão a partir de dados de campo foi $481 \mathrm{~m}^{3} \mathrm{~s}^{-1}$ (Figura 6).

Figura 6 - Medição e simulação de vazões e diagrama de dispersão correspondente ao ponto de monitoramento MP2 para calibração e validação

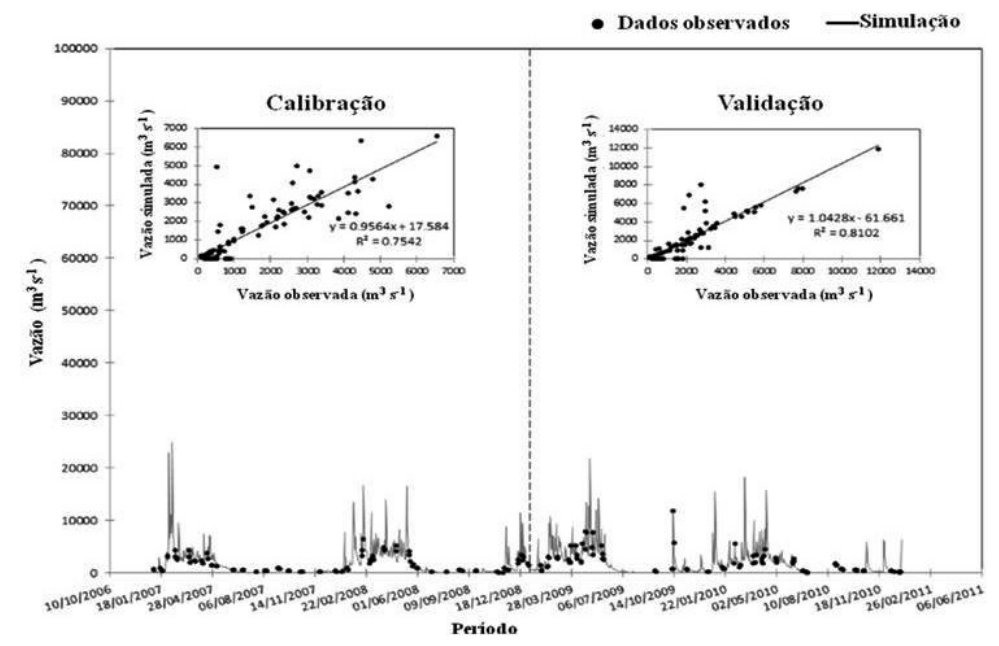

Fonte: Autores.

Na calibração manual dos pontos de monitorização MP1, MP2 e MP3, o declive $(\gamma)$ apresenta-se entre 0,9564 e 1,0926 e os valores do coeficiente de correlação $\left(R^{2}\right)$ variam entre 0,7542 e 0,9413 , para verificar se os valores são próximos de 1,0. No entanto, na Função Objetiva Normalizada (NOF) os valores são inferiores a 1,0 (entre 0,1530 e 0,6225) (Figuras 4, 5 e 6 e Tabela 4).

Tabela 4 - Calibração manual e validação da taxa de fluxo, aplicada à bacia Tocantins-Araguaia, nos pontos de monitoramento $\mathrm{MP}_{1}, \mathrm{MP}_{2}$ e $\mathrm{MP}_{3}$

\begin{tabular}{c|c}
\hline Pontos de monitoramento & Taxa de fluxo \\
\hline Calibração & Validação \\
Período de janeiro 2007 a dezembro de 2008 & $\begin{array}{c}\text { Período de janeiro 2009 a } \\
\text { dezembro de 2010 }\end{array}$ \\
\hline
\end{tabular}

\begin{tabular}{c|c|c|c|c|c|c|c|c}
\hline \multicolumn{1}{c}{ Parâmetros } \\
\hline & RMSE & NOF & $\gamma$ & R $^{2}$ & RMSE & NOF & $\gamma$ & $\mathrm{R}^{2}$ \\
\hline MP1 & 0.0002 & 0.1530 & 0.9964 & 0.8506 & 0.0006 & 0.6218 & 1.0334 & 0.8075 \\
\hline MP2 & 0.0008 & 0.6225 & 1.0926 & 0.9413 & 0.0004 & 0.3426 & 1.0079 & 0.9159 \\
\hline MP3 & 0.0014 & 0.3284 & 0.9564 & 0.7542 & 0.0004 & 0.1210 & 1.0428 & 0.8102 \\
\hline
\end{tabular}

Fonte: Autores. 
$\mathrm{Na}$ validação, o ponto de monitorização MP1 foi de $11,327 \mathrm{~m}^{3} \mathrm{~s}^{-1}$, sendo comparada com o valor da média da vazão a partir de dados de campo que foi de $11,907 \mathrm{~m}^{3} \mathrm{~s}^{-1}$ (Figura 4); em seguida, foi calculada a vazão média simulada no ponto de monitoramento MP2 que foi de $8,882 \mathrm{~m}^{3} \mathrm{~s}^{1}$, e comparada com o valor da média da vazão a partir de dados de campo que foi de $8,594 \mathrm{~m}^{3} \mathrm{~s}^{-1}$ (Figura 5); a vazão média simulada no ponto de monitoramento MP3 foi de 2,028 $\mathrm{m}^{3} \mathrm{~s}^{-1}$, colocada em comparação com o valor da média da vazão a partir de dados de campo que foi de $2,000 \mathrm{~m}^{3} \mathrm{~s}^{-1}$ (Figura 6).

$\mathrm{Na}$ validação manual dos pontos de monitoramento o MP1, MP2 e MP3, o declive $(\gamma)$ apresenta-se no intervalo de 1,0079 e 1,0428, e os valores dos coeficientes de correlação $\left(R^{2}\right)$ variam entre 0,8075 e 0,9159, sendo assim, ambos os valores $\left(\gamma\right.$ e R ${ }^{2}$ ) estão próximos de 1,0. No entanto, na Função Normalizada Objetiva (NOF) os valores são inferiores a 1,0 (entre 0,1210 e 0,6218) (Figuras 4, 5 e 6 e Tabela 4).

O modelo conseguiu representar com clareza a influência do aumento da precipitação sobre a vazão, comprovando que os pontos MP1 e MP2 se comportam de maneira direta e proporcional (Figura 7-a e Figura 7-b). Contudo, no ponto de monitoramento MP3 a vazão aumenta nos períodos mais chuvosos em proporções significantemente menores (Figura 7-c).

Figura 7 - (a) Valores simulados para a taxa de vazão e precipitação para o ponto de monitoramento MP1; (b) Valores simulados para a taxa de vazão e precipitação para o ponto de monitoramento MP2; (c) Valores simulados para a taxa de vazão e precipitação para o ponto de monitoramento MP3

(a)

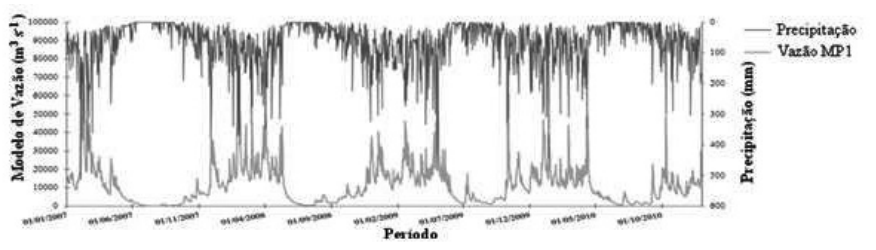

(b)

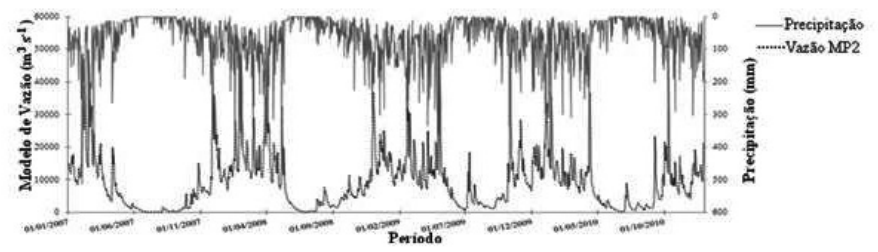

(c)

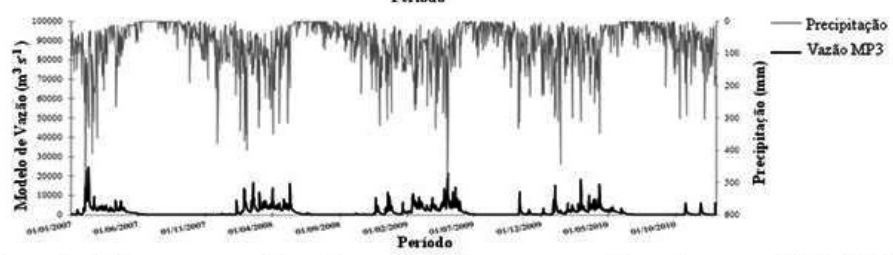

Fonte: Autores. 
A Figura 8 mostra a contribuição de cada ponto de monitoramento, destacando que em períodos de alta precipitação a cidade de Marabá e o reservatório da UHT tornam-se áreas de riscos à saúde ambiental, abrangendo os fatores físicos, químicos e biológicos externos às pessoas, e os fatores que impactam seus comportamentos em função da contribuição dos rios Tocantins e Araguaia (ponto MP2).

Figura 8 - Comparação de valores simulados para taxa de vazão e precipitação

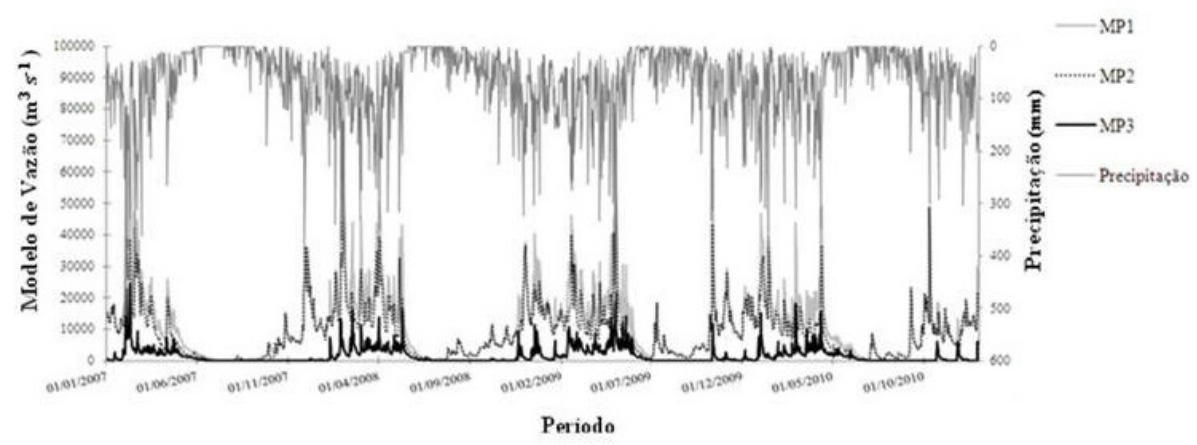

Fonte: Autores.

O modelo de vazão da bacia hidrográfica Tocantins-Araguaia, obtido a partir de dados reais, mostrou a grande probabilidade de ocorrência de desequilíbrios ambientais na região de estudo (Figura 9-a). Os cenários de gestão dos impactos gerados pela perda da cobertura do solo na margem da bacia indicam que pequenas mudanças no uso da terra (retirada de 10\% da cobertura vegetal) (Figura 9-b), elevam o volume de água e agravam a situação de risco e percentuais maiores (retirada de 50\% da cobertura vegetal) (Figura 9-c), podem provocar graves desastres ambientais na região de estudo.

Comparando os cenários (Figura 10), é possível identificar que pequenas alterações na paisagem, seja por ação antrópica e/ou natural, podem ser significativas para a gestão da vazão na bacia hidrográfica. 
Figura 9 - (a) Modelo de vazão com dados reais simulados da bacia hidrográfica Tocantins-Araguaia; (b) Cenário do modelo de vazão com perda de 10\% de cobertura vegetal; (c) Cenário do modelo de vazão com perda de $50 \%$ de cobertura vegetal

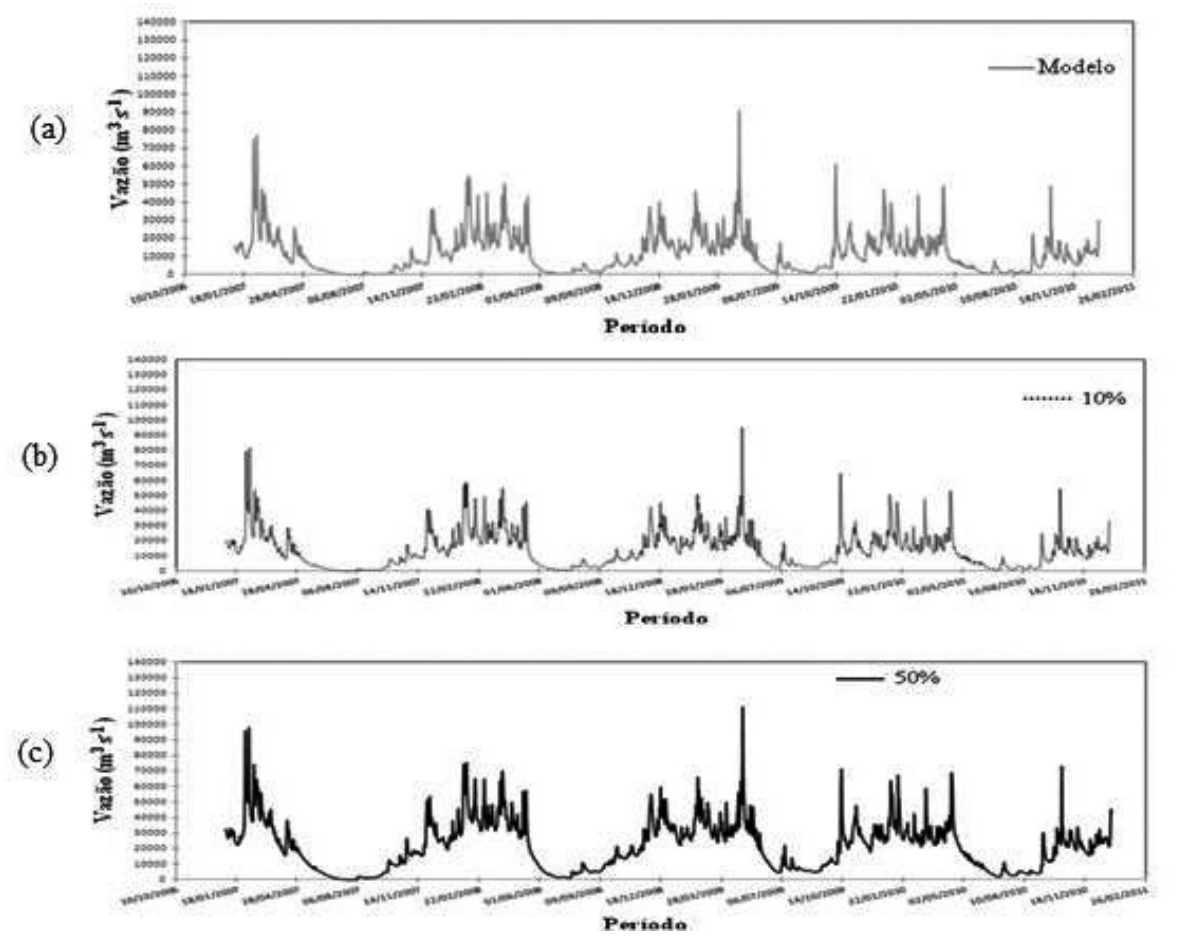

Fonte: Autores.

Figura 10 - Comparação do modelo de vazão com os cenários de perda de 10\% e $50 \%$ de cobertura vegetal da bacia hidrográfica Tocantins-Araguaia

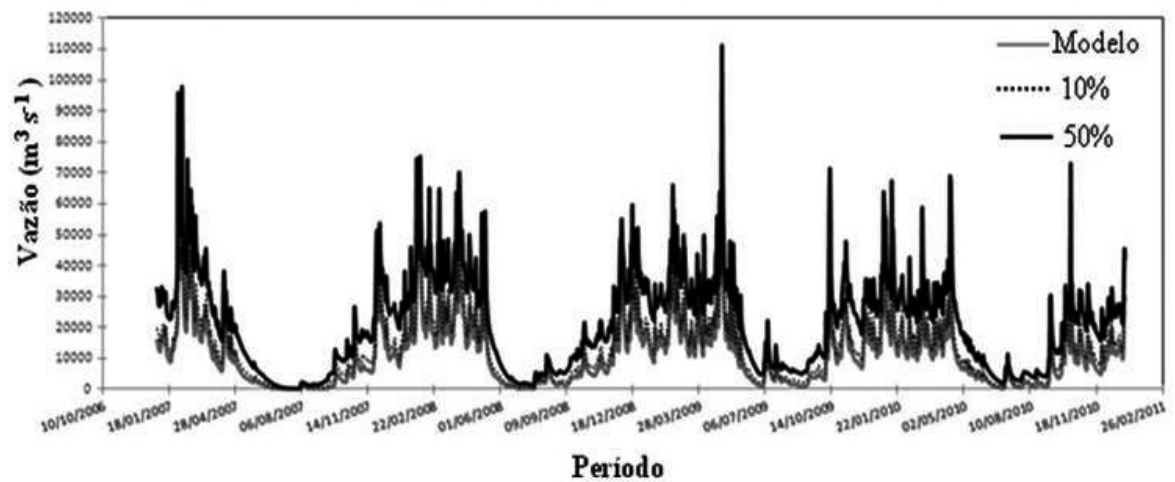

Fonte: Autores. 


\section{DISCUSSÃO}

3.1 O DESEMPENHO DO MODELO DE MONITORAMENTO E PREVISÃO DE VAZÃO DOS RIOS DA BACIA DO TOCANTINSARAGUAIA

O modelo apresentou valores aceitáveis quando comparados com as taxas de fluxo medidas tanto na calibração automática como na calibração manual (Figuras 4, 5, 6 e Tabela 4). Na validação, os resultados indicaram que o modelo foi capaz de reproduzir a série de taxa de fluxo que foi desconsiderada no período de calibração. Os resultados são significativos quando comparados com as taxas de fluxo observadas.

Nas saídas do modelo, considerando todo o período (2007-2010), foram simulados e comparados com as taxas de fluxo observadas (ver Figuras 4, 5 e 6). Estes resultados indicam que existe uma relação expressiva entre o modelo simulado e as observações, mostrando que o modelo é confiável e está diretamente proporcional à alteração na etapa de calibração e, por conseguinte, os resultados mostram que o modelo gerado simula a taxa média de fluxo diária aceitavelmente. Desse modo, o modelo mostrou que pode prever as taxas de fluxo dos rios da bacia hidrográfica Tocantins-Araguaia a partir dos índices de precipitação (Figura 7). Sendo possível perceber que o rio Itacaiúnas, Figura 7-c, apresenta pouca interferência na ocorrência de enchentes e deslizamentos de massa na região de estudo, sendo necessário para sua gestão apenas o aproveitamento da água em períodos de maior precipitação, ainda é importante enfatizar que estas medidas não resolveriam a problemática da enchente na região.

Contudo, no período de novembro a maio, a porção central, mais precisamente na cidade de Marabá (código "MARA", representando a cidade de Marabá na Figura 1), Figura 7-b, caracteriza-se como zona de risco de inundações e desequilíbrio ambiental, assim como a Usina Hidrelétrica de Tucuruí (Figura 7-a) também enfrenta problemas relacionados ao aumento no nível de água, sendo necessária, por vezes, a abertura de todas as comportas da barragem, o que provoca enchentes e graves danos ambientais a jusante (MANYARI, 2007). Esses acontecimentos podem ser acompanhados por noticiários locais, regionais e dependendo da gravidade emergencial decretada pode atingir níveis nacionais. Isto é comprovado, pois nos resultados do modelo é possível perceber os pontos de monitorização que sofrem maior influência da precipitação são MP1 e MP2, e, portanto, geram maiores escoamentos superficiais, alterando significativamente as taxas de fluxo. Desta forma, o ponto MP2 apresenta-se com maior influência 
das taxas de fluxo da bacia, sendo este representante dos rios Tocantins (rio principal) e Araguaia (afluente principal). Diante do exposto, é no ponto MP1, ver Figura 7-a, que descreve as taxas de fluxo da bacia hidrográfica TocantinsAraguaia, devido à proximidade com o exutório, apresentando-se com maiores picos de vazão em decorrência dos elevados índices de precipitação, ver Figura 8. Este resultado também indica que, em períodos de maior pluviosidade, poderia sofrer problemas ambientais como a erosão (CARVALHO; JONES; LIEBMANN, 2002), decorrente da dinâmica da bacia a montante.

\subsection{MUDANÇAS DE USO DA TERRA E GESTÃO DE CENÁRIOS}

Com correlações positivas entre a precipitação e a velocidade das taxas de fluxo modelada (Figura 9-a), os resultados indicam que os cenários com 10\% de desmatamento (Figura 9-b) alteram o escoamento superficial da água, ou seja, com perda de $10 \%$ da cobertura vegetal na bacia há influência significativa de chuvas e nas taxas de fluxo, no ponto MP1, causada pelo aumento do escoamento superficial e inundação de áreas próximas à barragem de UHT.

O cenário de $50 \%$ de retirada da cobertura vegetal indica emergência em toda margem da bacia, próximo à cidade de Marabá e a barragem de Tucuruí (Figura 9-c), visto que, de acordo com a carta de uso de solo (Tabela 2 e Figura 3), este fato demonstra um indicativo que no interior da bacia pode estar ocorrendo um aumento no uso indiscriminado do solo para agricultura e pecuária, associado com a carta de uso do solo (Figura 3), desencadeando intensa atividade erosiva na região. Em decorrência, tem aumentado o aporte de sedimentos provocando assoreamentos, o que pode estar favorecendo a ocorrência de enchentes e causando alterações ecológicas na bacia. Além dessas observações, a retirada dessa cobertura vegetal no modelo mostra que provavelmente toda área central da bacia poderá ser inundada pela força da água em períodos de alta precipitação.

A Figura 10 mostra com clareza o comportamento das taxas de fluxo diante da retirada da cobertura vegetal, proporcionando a comparação dos cenários. Desse modo, esta ferramenta apresenta-se como uma excelente estratégia de gestão, pois simula previsões de riscos ambientais provocados por ações antrópicas e/ou agentes naturais.

\section{CONCLUSÃO}

A criação do modelo hidrológico de previsão da bacia hidrográfica TocantinsAraguaia apresenta-se com grande representatividade no estudo das taxas de fluxo. 
O modelo digital de elevação do terreno forneceu um estudo mais detalhado e preciso da área da bacia, em função da divisão em sub-bacias e da criação das HRU. A análise do tipo, uso e ocupação do solo e da declividade permitiu a obtenção de informações necessárias para identificação do sentido e intensidade das taxas de fluxo, em cada ponto de observação. Desta forma, foi possível identificar que a maior parte de água excedente na saída da bacia é oriunda dos rios Tocantins e Araguaia e uma parte, em menor proporção, do rio Itacaiúnas.

A utilização de dados fluviométricos observados criou uma situação mais realística ao modelo, com representação de alta similaridade com as taxas de fluxo medidas nos pontos MP1, MP2 e MP3 e quando comparados os dados simulados das taxas de fluxo com dados de precipitação observados, no mesmo período, verificaram-se correlações positivas. Nesse sentido, foi possível perceber que durante os períodos de alta pluviosidade, entre novembro e maio, ocorre o aumento das taxas de fluxo e os riscos de acidentes ambientais.

Os resultados obtidos nos cenários também comprovam que o aumento dos níveis de precipitação-vazão é preocupante, principalmente quando estão associados ao desmatamento das margens do rio, haja vista que essas áreas poderão ser inundadas e/ou devastadas em maior proporção pela força da água. Esta capacidade do código SWAT em executar diferentes cenários de gestão o faz uma ferramenta poderosa para o processo de tomada de decisão.

Desse modo, seria muito importante que se fizessem maiores investimentos nestes estudos, haja vista que o Brasil é um país com bacias de grandes dimensões, poderia se utilizar essas tecnologias para minimizar o tempo e os custos em medição e monitoramento. A implementação do modelo SWAT na bacia do Tocantins-Araguaia foi a primeira tentativa de abordar a complexidade do sistema e forneceu estimativas razoáveis de tendências, onde há possibilidade de novos estudos devido à perda de vegetação que podem ser fator-chave para o processo de gestão de bacias hidrográficas e para a tomada de decisão.

\section{REFERÊNCIAS}

ANA. Diagnóstico do fluxo de sedimentos em suspensão na Bacia Araguaia-Tocantins. Brasília, DF: ANA, 2004.

ANA. Disponibilidade e demanda de recursos hídricos no Brasil. Brasília, DF: ANA, 2005.

ANA. Plano estratégico de recursos hídricos da bacia dos rios Tocantins e Araguaia: relatório síntese. Brasília, DF: ANA: SPR, 2009. 
ANA. Portal HidroWeb. Sistema Nacional de Informações sobre Recursos Hídricos, Brasília, DF. Disponível em: http://www.snirh.gov.br/hidroweb/ apresentacao. Acesso em: 17 out. 2012.

ANEEL. Atlas de energia elétrica do Brasil. Brasília, DF: ANEEL, 2002. Disponível em: www.aneel.gov.br/arquivos/PDF/livro_atlas.pdf. Acesso em: 17 out. 2012.

ARNOLD, J. G.; FOHRER, N. SWAT 2000: current capabilities and research opportunities in applied watershed modelling. Hydrological Processes, [S. l.], n. 19 , p. 563-572, 2005.

ARNOLD, J. G.; SRINIVASAN, R.; MUT'TIAH, R. S.; WILLIAMS, J. Large area hydrologic modeling and assessment part I: model development. Journal of the American Water Resources Association, [S. l.], n. 34, p. 73-89, 1998.

BERTONI, J.; LOMBARDI NETO, F. Conservação do Solo. São Paulo: Ícone Editora, 1990. 355 p.

CARVALHO, L. M. V.; JONES, C.; LIEBMANN, B. Extreme precipitation events in southern South America and large-scale convective patterns in the South Atlantic Convergence Zone. J. Climate, [S. l.], n. 15, p. 2377-2394, 2002. ELETROBRÁS, Usinas do Norte do Brasil - Sistema de Informação do Potencial Hidrelétrico Brasileiro - SIPOT. Brasília, DF: Eletrobrás, 2011.

ESBN. Soil Atlas of Europe. Luxembourg: ESDAC, 2005. 128 p.

ESWARAN, H.; RICE, T.; AHRENS, R.; STEWART, B. A. Soil Classification: a global desk reference. Boca Raton, FL: CRC Press, 2003. 272 p.

FITZHUGH, T. W.; MACKAY, D. S. Impacts of input parameter spatial aggregation on an agricultural nonpoint source pollution model. Journal of Hydrology, [S. l.], vol. 236, n. 1/2, p. 35-53, 2000.

GASSMAN, P. W.; REYES, M. R.; GREEN, C. H.; ARNOLD, J. G. The Soil and Water Assessment Tool: historical development, applications, and future research directions. American Society of Agricultural and Biological Engineers, [ $S$. l.], vol. 50, n. 4, p. 1211-1250, 2007.

HESSION, W. C.; SHANHOLTZ, V. O.; MOSTAGHIMI, S.; DILLAHA, T. A. Uncalibrated performance of the finite element storm hydrograph model. Transactions of ASAE, [S. l.], vol. 3, p. 777-783, 1994.

KORNECKI, T. S.; SABBAGH, G. J.; STORM, D. E. Evaluation of runoff, erosion and phosphorus modeling system-SIMPLE. Journal of the American Water Resources Association, [S. l.], vol. 4, p. 807-820, 1999. 
MACHADO, R. E. Simulação de escoamento e de produção de sedimentos em uma microbacia hidrográfica utilizando técnicas de modelagem e geoprocessamento. 2002. 152 f. Tese (Doutorado em Agricultura) Escola Superior de Agricultura Luiz de Queiroz, Universidade de São Paulo, Piracicaba, 2002.

MANYARI, W. V. A Impactos ambientais a jusante de hidrelétrica, o caso da usina de Tucuruí, PA. 2007. 211 f. Tese (Doutorado em Planejamento Estratégico) - Instituto Alberto Luiz Coimbra de Pós-Graduação e Pesquisa de Engenharia, Universidade Federal do Rio de Janeiro, Rio de Janeiro, 2007.

MMA. Região Hidrográfica Atlântico Leste. Brasília, DF: MMA/ANA, 2006. 156 p.

MORRIS, M. D. Factorial sampling plans for preliminary computational experiments. Technometrics, [S.l.], vol. 33, n. 2, p. 161-174, 1991.

NOGUEIRA, C. et al. Restricted-range fishes and the conservation of Brazilian freshwaters. Plos ONE, San Francisco, v. 5, n. 6, e11390, jun. 2010. Disponível em: http://dx.doi.org/10.1371/journal.pone.0011390. Acesso em: 17 out. 2012.

OAKES, H. The Soils of Turkey. Ankara: Dogus, 1954.

ONALP, A. Insaat Muhendislerine Geoteknik Bilgisi Cilt 1. Trabzon: Karadeniz Technical University, 1982.

OZTURK, I.; TANIK, A.; COKGOR, E. U.; GUREL, M.; MANTAS, E. P.; INSEL, G.; OZABALI, A. Buyuk Melen Watershed Integrated Protection and Water Management Master Plan. Istanbul: Istanbul Technical University, 2008.

PISINARAS, V.; PETALAS, C.; GIKAS, G. D.; GEMITZI, A.; TSIHRINTZIS, V. A. Hydrological and water quality modeling in a medium-sized basin using the Soil and Water Assessment Tool (SWAT). Desalination, [S. l.], vol. 250, n. 1, p. 274-286, 2010.

POLAT, A. H. Duzce ovasi sulama isletmesinde optimum su kullanim modelinin belirlenmesi uzerine bir arastirma. 2000. PhD (Thesis) - Gazi University, Institute of Science and Technology, Department of Construction, Ankara, 2000.

PREFEITURA MUNICIPAL DE MARABÁ. A cidade: histórico, localização, dados e economia. Prefeitura Municipal de Marabá, Marabá, 2010. Disponível em: http://www.maraba.pa.gov.br/a_cidade.htm. Acesso em: 14 out. 2010. 
PRIMAVESI, A. Manejo ecológico do solo: a agricultura em regiões tropicais. São Paulo: Editora Nobel, 1987. 549 p

PROJETO AVA MARABÁ. Relatório de Avaliação da Vulnerabilidade Ambiental. Marabá: PNUMA: MMA, 2006.

RAIOL, J. A. (coord). Perspectivas para o meio ambiente urbano: GEO Marabá-Pará. Belém: PNUMA: [s.n.], 2010. 136 p.

ROMANOWICZ, A. A.; VANCLOOSTER, M.; ROUNSEVELL, M.; LA JUNESSE, I. Sensitivity of the SWAT model to the soil and land use data parameterisation: a case study in the Thyle catchment, Belgium. Ecological Modelling, [S. l.], vol. 187, p. 27-39, 2005.

STRAUCH, M.; BAKKER, F. I.; ARAUJO, A.; LIMA, J. E. F. W.; LORZ, C.; MAKESCHIN, F. Assessing the hydrologic impact of conservation management practices for the Pipiripau River basin, Central Brazil, using SWAT. In: INTERNATIONAL SPECIALIZED CONFERENCE ON WATERSHED AND RIVER BASIN MANAGEMENT, 12., 2011, Recife. Proceedings [...]. Recife: IWA, 2011. p. 1-12.

TSIHRINTZIS, V. A.; HAMID, R. Runoff quality prediction from small urban catchments using SWMM. Hydrological Processes, [S. l.], vol. 12, p. 311-329, 1998.

TSIHRINTZIS, V. A.; SIDAN, C. B. Modeling urban storm water runoff using the Santa Barbara Method. Water Resources Management, [S. l.], vol. 12, p. 139-166, 1998.

VAN GRIENSVEN, A.; MEIXNER, T.; GRUNWALD, S.; BISHOP, T.; DILUZIO, M.; SRINIVASAN, R. A global sensitivity analysis tool for the parameters of multi-variable catchment models. Journal of Hydrology, [S. l.], n. 324, p. 10-23, 2006.

ZACHAR, D. Soil erosion development in soil science. New York: Elsevier Scientific, 1982.547 p. 